\title{
Vector invariants of symmetric groups in prime characteristic*
}

\section{S. A. STEPANOV}

Abstract - Let $R$ be a commutative ring with the unit element 1 and $S_{n}$ be the symmetric group of degree $n \geq 1$. Let $A_{m n}^{S_{n}}$ denote the subalgebra of invariants of the polynomial algebra

$$
A_{m n}=R\left[x_{11}, \ldots, x_{1 n} ; \ldots ; x_{m 1}, \ldots, x_{m n}\right]
$$

with respect to $S_{n}$. The classical result of $H$. Weyl implies that if every non-zero integer is invertible in $R$, then the algebra $A_{m n}^{S_{n}}$ is generated by the polarized elementary symmetric polynomials of degree at most $n$, no matter how large $m$ is. As it was recently shown by D. Richman, this result remains true under the condition that $\left|S_{n}\right|=n$ ! is invertible in $R$. On the other hand, if $R$ is a field of prime characteristic $p \leq n$, D. Richman proved that every system of $R$-algebra generators of $A_{m n}^{S_{n}}$ contains a generator whose degree is no less than $\max \{n,(m+p-n) /(p-1)\}$. The last result implies that the above Weyl bound on degrees of generators no longer holds when the characteristic $p$ of $R$ divides $\left|S_{n}\right|$. In general, it is proved that, for an arbitrary commutative ring $R$, the algebra $A_{m n}^{S_{n}}$ is generated by the invariants of degree at $\operatorname{most} \max \{n, m n(n-1) / 2\}$. The purpose of this paper is to give a simple arithmetical proof of the first result of $\mathrm{D}$. Richman and to sharpen his second result, again with the use of new arithmetical arguments. Independently, a similar refinement of Richman's lower bound was given by $\mathrm{G}$. Kemper on the basis of completely different considerations. A recent result of P. Fleischmann shows that the lower bound obtained in the paper is sharp if $m>1$ and $n$ is a prime power, $n=p^{\alpha}$.

\section{INTRODUCTION}

Let $m, n$ be positive integers, $R$ be a commutative ring with the unit element 1 , and

$$
A_{m n}=R\left[x_{11}, \ldots, x_{m 1} ; \ldots ; x_{1 n}, \ldots, x_{m n}\right]
$$

be the algebra of polynomials in $m n$ variables $x_{i j}$ over $R$. The symmetric group $S_{n}$ operates on the algebra $A_{m n}$ as a group of $R$-automorphisms by the rule

$$
\sigma\left(x_{i j}\right)=x_{i, \sigma(j)}, \quad \sigma \in S_{n} .
$$

Denote by $A_{m n}^{S_{n}}$ the subalgebra of invariants of the algebra $A_{m n}$ with respect to the group $S_{n}$ and define the polarized elementary symmetric polynomials $u_{r_{1}, \ldots, r_{m}} \in A_{m n}^{S_{n}}$ in $n$ vector variables

$$
\left(x_{11}, \ldots, x_{m 1}\right), \ldots,\left(x_{1 n}, \ldots, x_{m n}\right)
$$


by means of the formal identity

$$
\prod_{j=1}^{n}\left(1+x_{1 j} z_{1}+\ldots+x_{m j} z_{m}\right)=1+\sum_{1 \leq r_{1}+\ldots+r_{m} \leq n} u_{r_{1}, \ldots, r_{m}} z_{1}^{r_{1}} \ldots z_{m}^{r_{m}} .
$$

The elements of $A_{m n}^{S_{n}}$ are usually called the vector invariants of $S_{n}$. If $R$ is Noetherian, then it follows from the Hilbert-Noether finiteness theorem (see $[6,9])$ that $A_{m n}^{S_{n}}$ is a finitely generated commutative $R$-algebra and $A_{m n}$ is finitely generated as a module over $A_{m n}^{S_{n}}$. Moreover, if every non-zero integer is invertible in $R$, then the invariants $u_{r_{1}, \ldots, r_{m}}$ form a complete system of generators of $A_{m n}^{S_{n}}$ over $R$ (see [1], p. 9; [17], p:37). In other words, every element $u$ of the algebra $A_{m n}^{S_{n}}$ may be written as a polynomial in

$$
u_{r_{1}, \ldots, r_{m}}, \quad 1 \leq r_{1}+\ldots+r_{m} \leq n
$$

with coefficients in $R$. The above system of generating invariants contains $\left(\begin{array}{c}m+n \\ m\end{array}\right)-1$ elements connected with each other by different algebraic relations (see [4, p. 68] and [14]). This result was recently generalised by $\mathrm{D}$. Richman [11] as follows.

Theorem 1. Assume that $\left|S_{n}\right|=n$ ! is invertible in $R$. Then $A_{m n}^{S_{n}}$ is generated as an $R$ algebra by the polarized elementary symmetric polynomials

$$
u_{r_{1}, \ldots, r_{m}}, \quad 1 \leq r_{1}+\ldots+r_{m} \leq n
$$

of degree at most $n$.

In particular, if $R$ is a field of prime characteristic $p>n$, then $n$ ! is invertible in $R$, and we arrive at the following result.

Corollary 1. Let $R$ be a field. If $\operatorname{char} R=0$ or char $R=p>n$, then $A_{m n}^{S_{n}}$ is generated as an $R$-algebra by the polarized elementary symmetric polynomials

$$
u_{r_{1}, \ldots, r_{m}}, \quad 1 \leq r_{1}+\ldots+r_{m} \leq n .
$$

In this paper, we give a simple arithmetical proof of Theorem 1 based on polarisation of classical Waring's formulas [16] and closely related to the Weyl original proof [17] in the case where $R$ contains the field of rational numbers $\mathbf{Q}$.

The result of Theorem 1 can be easily extended as follows. Let $A=R\left[x_{1}, \ldots, x_{m}\right]$ be a finitely generated commutative $R$-algebra, $G$ be a finite group of $R$-algebra automorphisms of $A$, and $A^{G}$ be the subalgebra of invariants of $G$. If $z_{1}, \ldots, z_{m}$ are commuting indeterminates, then we set

$$
F\left(z_{1}, \ldots, z_{m}\right)=\prod_{\sigma \in G}\left(1+\sigma\left(x_{1}\right) z_{1}+\sigma\left(x_{2}\right) z_{2}+\ldots+\sigma\left(x_{m}\right) z_{m}\right) .
$$

If every non-zero integer is invertible in $R$, then it follows from the Noether theorem that $A^{G}$ is generated as an $R$-algebra by the coefficients of $F\left(z_{1}, \ldots, z_{m}\right)$. Theorem 1 and the standard arguments based on the use of the Reynolds operator and the Noether map (see [8]; [12], p. 63; [17], p. 275) lead to the following theorem. 
Theorem 2. If $|G|$ ! is invertible in $R$, then $A^{G}$ is generated as an R-algebra by the coefficients of $F\left(z_{1}, \ldots, z_{m}\right)$. In other words, $A^{G}$ is generated over $R$ by invariant polynomials in $x_{1}, \ldots, x_{m}$ of degree at most $|G|$.

The results of Theorems 1 and 3 provide us with an efficient algorithm to compute a complete system of generating polynomial invariants under the condition that $|G|$ ! is invertible in $R$. There is another constructive proof of Theorem 3 based on different arguments also ascending to Noether ([12], p. 29). The upper bound on the degrees of a set of generating polynomials for the algebra of invariants given by Theorem 3 is known as Noether's bound (see also [12], p. 28, and [13]).

If $\left|S_{n}\right|=n$ ! is invertible in $R$, then the upper bound on degrees of $R$-algebra generators of $A_{m n}^{S_{n}}$, stated in Theorem 1, is the best possible. In the case where $R$ is an arbitrary commutative ring, it is proved in [3] that the Weyl algebra $A_{m n}^{S_{n}}$ is generated over $R$ by polynomials of degree at most $\max \{n, m n(n-1) / 2\}$. A similar result was also obtained by Richman (see [11], Prop. 7). On the other hand, this paper gives the following lower bound.

Theorem 3. Let $\alpha$ be a positive integer, $S_{n}$ be the symmetric group of degree $n \geq 2$, and $R$ be a field of prime characteristic $p$. If $p^{\alpha}$ divides $n$, then every system of $R$-algebra generators of $A_{m n}^{S_{n}}$ contains a generator whose degree is no less than $\max \left\{n, m\left(p^{\alpha}-1\right)\right\}$.

This result sharpens the above mentioned Richman's lower bound in the case where $p$ is a divisor of $n$, and shows that Noether's upper bound is false if $n$ is not invertible in $R$. As it was recently proved by P. Fleischmann [5], the lower bound in Theorem 3 is exact if $n=p^{\alpha}$ and $m>1$.

The result of Theorem 3 can be easily extended as follows. Let $r$ be a positive integer that does not exceed $n$. In that case, the group $S_{r}$ is a subgroup of $S_{n}$ and therefore $A_{m n}^{S_{n}} \subseteq A_{m n}^{S_{r}}$. This observation and Theorem 3 applying to $S_{r}$ yield the following result.

Corollary 2. Let $\lambda$ and $r \leq n$ be positive integers, $S_{n}$ be the symmetric group of degree $n \geq 2$, and $R$ be a field of prime characteristic $p$. If $p^{\lambda}$ divides $r$, then in any system of $R$-algebra generators of $A_{m n}^{S_{n}}$ there exists a generator whose degree is no less than $\max \left\{n, m\left(p^{\lambda}-1\right)\right\}$.

Corollary 3. Let $S_{n}$ be the symmetric group of degree $n \geq 2$ and $R$ be a commutative ring with the unit element 1 . If $p$ is a prime divisor of $n !$ which is not invertible in $R$, then every system of $R$-algebra generators of $A_{m n}^{S_{n}}$ contains a generator whose degree is no less than $\max \{n, m(p-1)\}$.

If $R=\mathbf{Z}$ is the ring of integers, then we can use the well-known results on the distribution of primes in short intervals to get an universal lower bound in terms of $m$ and $n$.

Corollary 4. Let $R=\mathbf{Z}$ be the ring of integers and $S_{n}$ be the symmetric group of degree $n \geq 2$. Then every system of $R$-algebra generators of $A_{m n}^{S_{n}}$ contains a generator whose degree is no less than $\max \left\{n, c_{n} m(n-1)\right\}$, where $c_{n}=1 / 2$ for every $n \geq 2 ;$ moreover, $c_{n}=5 / 6$ for every $n \geq 25$, and $c_{n} \rightarrow 1$ as $n \rightarrow \infty$. In particular, if $n$ is a prime number, then every 
system of R-algebra generators of $A_{m n}^{S_{n}}$ contains a generator whose degree is no less than $\max \{n, m(n-1)\}$.

A similar result holds in the case where $R$ is the ring $\mathbf{Z}_{K}$ of integers of a number field $K$.

\section{GENERATING INVARIANTS OF THE SYMMETRIC GROUP $S_{n}$}

Let $S_{n}$ be the symmetric group of degree $n \geq 1$ that operates on the $R$-algebra

$$
A_{m n}=R\left[x_{11}, \ldots, x_{m 1} ; \ldots ; x_{1 n}, \ldots, x_{m n}\right]
$$

as a group of $R$-automorphisms, $A_{m n}^{S_{n}}$ be the subalgebra of invariants of the algebra $A_{m n}$, and

$$
u_{r_{1}, \ldots, r_{m}}, \quad 1 \leq r_{1}+\ldots+r_{m} \leq n
$$

be the polarized elementary symmetric polynomials in $A_{m n}^{S_{n}}$.

Let $v_{s_{1}}, \ldots, s_{m}$ be an invariant polynomial in $A_{m n}^{S_{n}}$ of the form

$$
v_{s_{1}, \ldots, s_{m}}=\sum_{j=1}^{n} x_{1 j}^{s_{1}} \ldots x_{m j}^{s_{m}} .
$$

If $m=1$, then according to the well-known Waring formula (see [16], p. 13, and [2], p. 99)

$$
v_{s}=\sum_{j=1}^{n} x_{j}^{s}=\sum_{k_{1}+2 k_{2}+\ldots+n k_{n}=s} c\left(k_{1}, \ldots, k_{n}\right) u_{1}^{k_{1}} \ldots u_{n}^{k_{n}},
$$

where $c_{k_{1}, \ldots, k_{n}}$ are integers of the form

$$
c\left(k_{1}, \ldots, k_{n}\right)=(-1)^{k_{2}+2 k_{3}+\ldots+(n-1) k_{n}} \frac{\left(k_{1}+\ldots+k_{n}-1\right) !}{k_{1} ! \ldots k_{n} ! s} .
$$

The following result can be considered as a generalisation of the Waring formula to the case where $m>1$ (see also [14], [15]).

Proposition 1. Let $s_{1}, \ldots, s_{m}$ be non-negative integers,

$$
v_{s_{1}, \ldots, s_{m}}=\sum_{j=1}^{n} x_{1 j}^{s_{1}} \ldots x_{m j}^{s_{m}}
$$

be the polynomial in $A_{m n}^{S_{n}}$ of degree $s=s_{1}+\ldots+s_{m}$, and

$$
u_{r_{1}, \ldots, r_{m}}, \quad 1 \leq r_{1}+\ldots+r_{m} \leq n
$$

be the polarized elementary symmetric polynomials of the vectors

$$
\left(x_{1 j}, \ldots, x_{m j}\right), \quad 1 \leq j \leq n .
$$


For non-negative integers $k_{1}, \ldots, k_{n}$ and $k_{1 v}, \ldots, k_{m v}$ such that

$$
\begin{aligned}
k_{1}+2 k_{2}+\ldots+n k_{n} & =s, \\
k_{1 v}+\ldots+k_{m v} & =v k_{v}, \quad 1 \leq v \leq n,
\end{aligned}
$$

let

$$
w_{k_{1 v}, \ldots, k_{m v}}=\sum_{A_{m, n}} \frac{k_{v} !}{l_{v 1} ! \ldots l_{v k_{v}} !} \prod_{\mu=1}^{k_{v}} u_{r_{1 \mu}, \ldots, r_{m \mu}}^{l_{v \mu}},
$$

where the sum is over the set $A_{m, n}$ of all non-negative integers $r_{1 \mu}, \ldots, r_{m \mu}$ and $l_{v 1}, \ldots, l_{v k_{v}}$ such that

$$
\begin{aligned}
r_{i 1} l_{v 1}+\ldots r_{i k_{v}} l_{v k_{v}} & =k_{i v}, \\
l_{v 1}+\ldots+l_{v k_{v}} & =k_{v}, \\
r_{1 \mu}+\ldots+r_{m \mu} & =v
\end{aligned}
$$

for $1 \leq i \leq m, 1 \leq \mu \leq k_{v}, 1 \leq v \leq n$.

Then

$$
v_{s_{1}, \ldots, s_{m}}=\frac{s_{1} ! \ldots s_{m} !}{s !} \sum_{k_{1}+2 k_{2}+\ldots+n k_{n}=s} c\left(k_{1}, \ldots, k_{n}\right) \sum_{B_{m, n}} \prod_{v=1}^{n} w_{k_{1 v}, \ldots, k_{m v}},
$$

where the inner sum is over the set $B_{m, n}$ of all non-negative integers $k_{i 1}, \ldots, k_{i n}$ satisfying the relations

$$
\begin{aligned}
k_{i 1}+\ldots+k_{i n} & =s_{i}, \\
k_{1 v}+\ldots+k_{m v} & =v k_{v}
\end{aligned}
$$

for $1 \leq i \leq m, 1 \leq v \leq n$.

Proof. In (1) we set

$$
x_{j}=x_{1 j} z_{1}+\ldots+x_{m j} z_{m}, \quad 1 \leq j \leq n .
$$

Since

$$
\left(x_{1 j} z_{1}+\ldots+x_{m j} z_{m}\right)^{s}=\sum_{s_{1}+\ldots+s_{m}=s} \frac{s !}{s_{1} ! \ldots s_{m} !} x_{1 j}^{s_{1}} \ldots x_{m j}^{s_{m}} z_{1}^{s_{1}} \ldots z_{m}^{s_{m}}
$$

we have

$$
\sum_{j=1}^{n}\left(x_{1 j} z_{1}+\ldots+x_{m j} z_{m}\right)^{s}=\sum_{s_{1}+\ldots+s_{m}=s} \frac{s !}{s_{1} ! \ldots s_{m} !}\left(\sum_{j=1}^{n} x_{1 j}^{s_{1}} \ldots x_{m j}^{s_{m}}\right) z_{1}^{s_{1}} \ldots z_{m}^{s_{m}} .
$$

On the other hand,

$$
\sum_{1 \leq j_{1}<\ldots<j_{v} \leq n} \prod_{k=1}^{v}\left(x_{1 j_{k}} z_{1}+\ldots+x_{m j_{k}} z_{m}\right)=\sum_{r_{1}+\ldots+r_{m}=v} u_{r_{1}, \ldots, r_{m}} z_{1}^{r_{1}} \ldots z_{m}^{r_{m}}
$$


and hence, in view of (1),

$$
\begin{aligned}
\sum_{j=1}^{n}\left(x_{1 j} z_{1}+\ldots+x_{m j} z_{m}\right)^{s} & \\
& =\sum_{k_{1}+2 k_{2}+\ldots+n k_{n}=s} c\left(k_{1}, \ldots, k_{n}\right) \prod_{v=1}^{n}\left(\sum_{r_{1}+\ldots+r_{m}=v} u_{r_{1}, \ldots, r_{m}} z_{1}^{r_{1}} \ldots z_{m}^{r_{m}}\right)^{k_{v}}
\end{aligned}
$$

As a result we find that

$$
\begin{gathered}
\sum_{s_{1}+\ldots+s_{m}=s} \frac{s !}{s_{1} ! \ldots s_{m} !}\left(\sum_{j=1}^{n} x_{1 j}^{s_{1}} \ldots x_{m j}^{s_{m}}\right) z_{1}^{s_{1}} \ldots z_{m}^{s_{m}} \\
\sum_{k_{1}+2 k_{2}+\ldots+n k_{n}=s} c\left(k_{1}, \ldots, k_{n}\right) \prod_{v=1}^{n}\left(\sum_{r_{1}+\ldots+r_{m}=v} u_{r_{1}, \ldots, r_{m}} z_{1}^{r_{1}} \ldots z_{m}^{r_{m}}\right)^{k_{v}}
\end{gathered}
$$

Now since

$$
\left(\sum_{r_{1}+\ldots+r_{m}=v} u_{r_{1}, \ldots, r_{m}} z_{1}^{r_{1}} \ldots z_{m}^{r_{m}}\right)^{k_{\nu}}=\sum_{k_{1 v}+\ldots+k_{m v}=v k_{v}} w_{k_{1 v}, \ldots, k_{m v}} z_{1}^{k_{1 v}} \ldots z_{m}^{k_{m v}}
$$

where

$$
w_{k_{1 v} \ldots, k_{m v}}=\sum_{C_{m, v}} \frac{k_{v} !}{l_{v 1} ! \cdots l_{v k_{v}} !} \prod_{\mu=1}^{k_{v}} u_{r_{1 \mu}, \ldots, r_{m \mu}}^{l_{v \mu}}
$$

and the summation is over the set $C_{m, v}$ of all non-negative integers $r_{1 \mu}, \ldots, r_{m \mu}$ and $l_{v 1}, \ldots, l_{v k_{v}}$ such that

$$
\begin{aligned}
r_{i 1} l_{v 1}+\ldots+r_{i s_{v}} l_{v k_{v}} & =k_{i v}, & 1 \leq i \leq m \\
r_{1 \mu}+\ldots+r_{m \mu} & =v, & 1 \leq \mu \leq k_{v} \\
l_{v 1}+\ldots+l_{v k_{v}} & =k_{v}, &
\end{aligned}
$$

we find that

$$
\begin{aligned}
& \sum_{s_{1}+\ldots+s_{m}=s} \frac{s !}{s_{1} ! \ldots s_{m} !}\left(\sum_{j=1}^{n} x_{1 j}^{s_{1}} \ldots x_{m j}^{s_{m}}\right) z_{1}^{s_{1}} \ldots z_{m}^{s_{m}} \\
& \quad=\sum_{s_{1}+\ldots+s_{m}=s}\left(\sum_{k_{1}+2 k_{2}+\ldots+n k_{n}=s} c\left(k_{1}, \ldots, k_{n}\right) \sum_{B_{m, n}} \prod_{v=1}^{n} w_{k_{1 v}, \ldots, k_{m v}}\right) z_{1}^{s_{1}} \ldots z_{m}^{s_{m}}
\end{aligned}
$$

where the summation in the last sum is over the set $B_{m, n}$ of all non-negative integers $k_{i 1}, \ldots, k_{i v}, i=1, \ldots, m$ such that

$$
\begin{aligned}
k_{i 1}+\ldots+k_{i v} & =s_{i} \\
k_{1 v}+\ldots+k_{m v} & =v k_{v}
\end{aligned}
$$


for $1 \leq i \leq m, 1 \leq v \leq n$.

Thus we arrive at the relation

$$
\sum_{j=1}^{n} x_{1 j}^{s_{1}} \ldots x_{m j}^{s_{m}}=\frac{s_{1} ! \ldots s_{m} !}{s !} \sum_{k_{1}+2 k_{2}+\ldots+n k_{n}=s} c\left(k_{1}, \ldots, k_{n}\right) \sum_{B_{m, n}} \prod_{v=1}^{n} w_{k_{1 v}, \ldots, k_{m v}},
$$

which proves the proposition.

$$
\begin{aligned}
& \text { If } s=s_{1}+\ldots+s_{m} \leq n+1 \text {, then } \\
& \qquad v_{s_{1}, \ldots, s_{m}}=\frac{s_{1} ! \ldots s_{m} !}{s !} \sum_{k_{1}+2 k_{2}+\ldots+n k_{n}=s} c\left(k_{1}, \ldots, k_{n}\right) \sum_{B_{m, n}} \prod_{v=1}^{n} w_{k_{1 v}, \ldots, k_{m v}}
\end{aligned}
$$

is a polynomial in $u_{r_{1}, \ldots, r_{m}}, 1 \leq r_{1}+\ldots+r_{m} \leq n$, with rational coefficients whose denominators are not divisible by any prime $p \geq n+1$. As a consequence of this observations we get the following result.

Corollary 5. If $n$ ! is invertible in $R$ and $s=s_{1}+\ldots+s_{m} \leq n+1$, then

$$
v_{s_{1}, \ldots, s_{m}}=\sum_{j=1}^{n} x_{1 j}^{s_{1}} \ldots x_{m j}^{s_{m}}
$$

is a polynomial over $R$ in $u_{r_{1}, \ldots, r_{m}}, 1 \leq r_{1}+\ldots+r_{m} \leq n$, of degree at most $n$.

Now we show that if $n$ ! is invertible in $R$, then any vector invariant in $A_{m n}^{S_{n}}$ can be represented as a polynomial over $R$ in the invariants $v_{s_{1}, \ldots, s_{m}}$.

Proposition 2. Let $f$ be a monomial in $A_{m n}$ and

$$
v=\sum_{u \in\left\{\sigma(f) \mid \sigma \in S_{n}\right\}} u .
$$

If $n$ ! is invertible in $R$, then $v$ is a polynomial over $R$ in the invariants

$$
v_{s_{1}, \ldots, s_{m}}=\sum_{j=1}^{n} x_{1 j}^{s_{1}} \ldots x_{m j}^{s_{m}},
$$

where $s_{1}, \ldots, s_{m}$ are non-negative integers satisfying the condition

$$
0 \leq s_{1}+\ldots+s_{m} \leq \operatorname{deg} f \text {. }
$$

Proof. We represent $f$ in the form $f=f_{1} \ldots f_{n}$, where each $f_{j}$ is a monomial in $R\left[x_{1 j}, \ldots, x_{m j}\right]$. We set

$$
d(f)=\max _{1 \leq j \leq n}\left(\operatorname{deg} f_{j}\right)
$$

and prove the assertion by induction on $\delta(f)=\operatorname{deg} f-d(f)$. Suppose at first that $\delta(f)=0$. Then $f=f_{j}=x_{1 j}^{s_{1}} \ldots x_{m j}^{s_{m}}$, where $j \in\{1,2, \ldots, n\}$ and $s_{1}, \ldots, s_{m}$ are non-negative integers with the condition $s_{1}+\ldots+s_{m}=\operatorname{deg} f$, and

$$
v=\sum_{u \in\left\{\sigma(f) \mid \sigma \in S_{n}\right\}} u=\sum_{j=1}^{n} x_{1 j}^{s_{1}} \ldots x_{m j}^{s_{m}} .
$$


Suppose now that $\delta(f)>0$ and let $j \in\{1,2, \ldots, n\}$ be such that

$$
d(f)=\operatorname{deg} f_{j}<\operatorname{deg} f .
$$

we define $v_{j}$ and $v_{j}^{\prime}$, setting

$$
v_{j}=\sum_{u \in\left\{\sigma\left(f_{j}\right) \mid \sigma \in S_{n}\right\}} u, \quad v_{j}^{\prime}=\sum_{u^{\prime} \in\left\{\sigma\left(f / f_{j}\right) \mid \sigma \in S_{n}\right\}} u^{\prime} .
$$

The induction hypothesis implies that $v_{j}$ and $v_{j}^{\prime}$ are polynomials in

$$
v_{s_{1}, \ldots, s_{m}}, \quad 0 \leq s_{1}+\ldots+s_{m} \leq \operatorname{deg} f .
$$

For every $\tau \in S_{n}$, we define $U_{\tau}$ to be the set of all pairs $\left(u, u^{\prime}\right)$ such that

$$
u \in\left\{\sigma\left(f_{j}\right) \mid \sigma \in S_{n}\right\}, \quad u^{\prime} \in\left\{\sigma\left(f / f_{j}\right) \mid \sigma \in S_{n}\right\}, \quad u u^{\prime}=\tau(f)
$$

and note that the map

$$
U_{i d} \rightarrow U_{\tau}, \quad\left(u, u^{\prime}\right) \rightarrow\left(\tau(u), \tau\left(u^{\prime}\right)\right)
$$

is a bijection. Thus $\left|U_{\tau}\right|=\left|U_{i d}\right|$ for all $\tau \in S_{n}$. Note also that $d\left(u u^{\prime}\right) \geq d(f)$ for all $u \in$ $\left\{\sigma\left(f_{j}\right) \mid \sigma \in S_{n}\right\}$ and $u^{\prime} \in\left\{\sigma\left(f / f_{j}\right) \mid \sigma \in S_{n}\right\}$, where the equality is attained if and only if $u u^{\prime} \in\left\{\sigma(f) \mid \sigma \in S_{n}\right\}$. Therefore,

$$
v_{j} v_{j}^{\prime}=\left|U_{i d}\right| \sum_{u \in\left\{\sigma(f) \mid \sigma \in S_{n}\right\}} u+\sum_{f^{\prime}: \operatorname{deg} f^{\prime}=\operatorname{deg} f, d\left(f^{\prime}\right)>d(f)} \sum_{u \in\left\{\sigma\left(f^{\prime}\right) \mid \sigma \in S_{n}\right\}} u .
$$

By the induction hypothesis, the invariant

$$
v_{j} v_{j}^{\prime}-\left|U_{i d}\right| \sum_{u \in\left\{\sigma(f) \mid \sigma \in S_{n}\right\}} u=\sum_{f^{\prime}: \operatorname{deg} f^{\prime}=\operatorname{deg} f, d\left(f^{\prime}\right)>d(f)} \sum_{u \in\left\{\sigma\left(f^{\prime}\right) \mid \sigma \in S_{n}\right\}} u
$$

is a polynomial over $R$ in $v_{s_{1}, \ldots, s_{m}}, 0 \leq s_{1}+\ldots+s_{m} \leq \operatorname{deg} f$. The cardinality of $U_{i d}$ does not exceed the cardinality of $\left\{\sigma\left(f_{j}\right) \mid \sigma \in S_{n}\right\}$ and the last cardinality does not exceed the cardinality of $\left\{x_{1 j}^{s_{1}} \ldots x_{m j}^{s_{m}} \mid 1 \leq j \leq n\right\}$, therefore $1 \leq\left|U_{i d}\right| \leq n$. Since $n$ ! is invertible in $R$, we conclude that

$$
\sum_{u \in\left\{\sigma(f) \mid \sigma \in S_{n}\right\}} u
$$

is a polynomial over $R$ in $v_{s_{1}, \ldots, s_{m}}, 0 \leq \sigma_{1}+\ldots+\sigma_{m} \leq \operatorname{deg} f$. This completes the proof.

Let $S_{n}$ be the symmetric group of degree $n \geq 2$ and

$$
v_{s_{1}, \ldots, s_{m}}=\sum_{j=1}^{n} x_{1 j}^{s_{1}} \ldots x_{m j}^{s_{m}}
$$

be a homogeneous polynomial in $A_{m n}^{S_{n}}$ of degree $s_{1}+\ldots+s_{m} \geq 1$. Let $R$ be a field of prime characteristic $p$ dividing $n$. The following result shows that Weyl's bound fails to be correct over $R$ for $m>n \geq 2$. 
Proposition 3. Let $R$ be a field of prime characteristic $p$ and $S_{n}$ be the symmetric group of degree $n \geq 2$. If $p$ divides $n$, and $m>n$, then the element $v_{11 \ldots 11} \in A_{m n}^{S_{n}}$ cannot be expressed as a polynomial over $R$ in the invariants

$$
u_{r_{1}, \ldots, r_{m}}, \quad 1 \leq r_{1}+\ldots+r_{m} \leq n .
$$

Proof. Assume, for a contradiction, that $v_{11 \ldots 11}$ is expressible as a polynomial over $R$ in $u_{r_{1}, \ldots, r_{m}}, 1 \leq r_{1}+\ldots+r_{m} \leq n$, and write $v_{11 \ldots 11}$ in the form

$$
v_{11 \ldots 11}=\sum_{s_{1}+2 s_{2}+\ldots+n s_{n}=m} a_{s_{1}, \ldots, s_{n}} \sum_{R_{m}\left(s_{1}, \ldots, s_{n}\right)} \prod_{\nu=1}^{n} \prod_{\sigma_{v}=1}^{s_{v}} u_{r_{1 \sigma_{v}}, \ldots, r_{m \sigma_{v}}}
$$

with some $a_{s_{1}, \ldots, s_{n}} \in R$, where $R_{m}\left(s_{1}, \ldots, s_{n}\right)$ is the set of all non-negative integers $r_{i} j, i=$ $1, \ldots, m, j=1, \ldots, s_{v}, v=1, \ldots, n$ such that

$$
\begin{aligned}
r_{1 \sigma_{v}}+\ldots+r_{m \sigma_{v}} & =v, \quad 1 \leq \sigma_{v} \leq s_{v}, \quad 1 \leq v \leq n, \\
r_{i \sigma_{1}}+\ldots+r_{i \sigma_{n}} & =1, \quad 1 \leq i \leq m .
\end{aligned}
$$

Without loss of generality we may assume that if $k \leq n$ is the smallest positive integer such that $s_{k} \geq 1$, then

$$
r_{1 \sigma_{k}}= \begin{cases}1 & \text { if } \sigma_{k}=1 \\ 0 & \text { if } 2 \leq \sigma_{k} \leq s_{k}\end{cases}
$$

Differentiating the above equality with respect to $x_{11}$ and taking into account that

$$
\frac{\partial u_{r_{1}, \ldots, r_{m}}}{\partial x_{11}}= \begin{cases}0 & \text { if } r_{1}=0 \\ u_{0, r_{2}, \ldots, r_{m}}^{(1,0, \ldots, 0)} & \text { if } r_{1}=1\end{cases}
$$

where $u_{0, r_{2}, \ldots, r_{m}}^{(1,0, \ldots, 0)}$ is the corresponding elementary symmetric polynomial of vectors $\left(x_{2 j}, \ldots, x_{m j}\right), 1 \leq j \leq n$, we obtain

$$
x_{21} \ldots x_{m 1}=\sum_{s_{1}+2 s_{2}+\ldots+n s_{n}=m} a_{s_{1}, \ldots, s_{n}} \sum_{j=1}^{n} \Psi_{s_{1}, \ldots, s_{n}}^{(j)},
$$

where

$$
\Psi_{s_{1}, \ldots, s_{n}}^{(j)}=\sum_{R_{m}\left(s_{1}, \ldots, s_{n}\right)} u_{0, r_{2 \sigma_{k}}, \ldots, r_{m \sigma_{k}}}^{(1,0, \ldots, 0)} \prod_{\sigma_{k}=2}^{s_{k}} u_{0, r_{2 \sigma_{k}}, \ldots, r_{m \sigma_{k}}} \prod_{v=1, v \neq k}^{n} \prod_{\sigma_{v}=1}^{s_{v}} u_{0, r_{2 \sigma_{v}} \ldots, r_{m \sigma_{v}}} .
$$

We denote by $\omega_{0, r_{2}, \ldots, r_{m}}$ the value of $u_{0, r_{2}, \ldots, r_{m}}$ at the point

$$
\left(x_{11}, \ldots, x_{m 1} ; \ldots ; x_{1 n}, \ldots, x_{m n}\right)=(1, \ldots, 1 ; \ldots ; 1 \ldots, 1) .
$$

Since $m>n \geq 2$, each binary sequence $\left(0, r_{2}, \ldots, r_{m}\right)$ encountered in the last equality contains $l$ non-zero elements for some $1 \leq l \leq n$. In that case

$$
\omega_{0, r_{2}, \ldots, r_{m}}=n(n-1) \ldots(n-l+1)
$$


and setting

$$
x_{11}=\ldots=x_{m 1}=\ldots=x_{1 n}=\ldots=x_{m n}=1
$$

in (2), we arrive at the relation

$$
1=n \sum_{s_{1}+2 s_{2}+\ldots+n s_{n}=m} b_{s_{1}, \ldots, s_{n}}
$$

which is impossible in $R$ for any prime $p$ dividing $n$. This completes the proof.

\section{PROOF OF THEOREM 1}

Let $S_{n}$ be the symmetric group of degree $n$. Suppose that $f$ is a monomial in $A_{m n}$ and $w \in A_{m n}^{S_{n}}$ is a polynomial invariant of $S_{n}$. Since $\sigma(w)=w$ for any $\sigma \in S_{n}$, the polynomials $w$ and $\sigma(w)$ have equal coefficients. This shows that every polynomial invariant of $S_{n}$ is an $R$-linear combination of the invariants

$$
v=\sum_{u \in\left\{\sigma(f) \mid \sigma \in S_{n}\right\}} u
$$

where $f$ varies over the monomials which appear in $w$.

Let $\left(i_{1}, i_{2}, \ldots, i_{\mu}\right)$ be a sequence of elements $i_{1}, i_{2}, \ldots, i_{\mu} \in\{1,2, \ldots, n\}$. At first we prove that every invariant $w_{\mu}$ of the form

$$
w_{\mu}=\sum_{j=1}^{n} x_{i_{1}, j} \ldots x_{i_{\mu}, j}
$$

is a polynomial over $R$ in polarized elementary symmetric polynomials

$$
u_{r_{1}, \ldots, r_{m}}, \quad 1 \leq r_{1}+\ldots+r_{m} \leq n .
$$

If $\mu \leq n+1$, then the assertion of Theorem 1 follows from Proposition 1 . Assume now that $\mu>n+1$ and proceed the proof by induction on $\mu$. Let

$$
\tilde{x}_{i_{s}, j}= \begin{cases}x_{i_{s}, j} & \text { if } s \leq n \\ x_{i_{n+1}, j} x_{i_{n+2}, j} \ldots x_{i_{\mu+1}, j} & \text { if } s=n+1\end{cases}
$$

for $j=1,2, \ldots, n$, and write

$$
w_{\mu+1}=\sum_{j=1}^{n} \tilde{x}_{i_{1}, j} \ldots \tilde{x}_{i_{n}, j} \tilde{x}_{i_{n+1}, j}
$$

Let

$$
\tilde{A}_{m n}=R\left[\tilde{x}_{11}, \ldots, \tilde{x}_{m 1} ; \ldots ; \tilde{x}_{1 n}, \ldots, \tilde{x}_{m n}\right]
$$

and let $\tilde{A}_{m n}^{S_{n}}$ be the subalgebra of invariants of $\tilde{A}_{m n}$. It follows from Corollary 5 that $w_{\mu+1}$ is a polynomial over $R$ in the polarized elementary symmetric polynomials

$$
\tilde{u}_{r_{1}, \ldots, r_{m}} \in \tilde{A}_{m n}, \quad 1 \leq r_{1}+\ldots+r_{m} \leq n
$$


Since every such polynomial $\tilde{u}_{r_{1}, \ldots, r_{m}}$ has the form

$$
\tilde{u}_{r_{1}, \ldots, r_{m}}=\sum_{\tilde{u} \in\{\tau(\tilde{f}) \mid \tau \in G\}} \tilde{u}
$$

for some monomial $\tilde{f} \in \tilde{A}_{m n}$ of degree at most $n$, by Proposition 1 it can be written as a polynomial over $R$ in the invariants

$$
\tilde{v}_{s_{1}, \ldots, s_{m}}=\sum_{j=1}^{n} \tilde{x}_{1 j}^{s_{1}} \ldots \tilde{x}_{m j}^{s_{m}}
$$

of degree at most $n$. Moreover, each $\tilde{v}_{s_{1}, \ldots, s_{m}}$ has the form

$$
\tilde{v}_{s_{1}, \ldots, s_{m}}=\sum_{j=1}^{n} x_{1 j}^{t_{1}} \cdots x_{m j}^{t_{j}}
$$

where $1 \leq t_{1}+\ldots+t_{m} \leq \mu$. The induction hypothesis implies that every invariant $\tilde{v}_{s_{1}, \ldots, m}$ is a polynomial over $R$ in

$$
u_{r_{1}, \ldots, r_{m}}, \quad 1 \leq r_{1}+\ldots+r_{m} \leq n,
$$

therefore $w_{\mu+1}$ is also a polynomial over $R$ in the polarized elementary symmetric polynomials $u_{r_{1}, \ldots, r_{m}}, 1 \leq r_{1}+\ldots+r_{m} \leq n$.

To complete the proof, we note now that every element $v \in A_{m n}^{S_{n}}$ can be written, in view of Proposition 2, as a polynomial over $R$ in the invariants $w_{\mu}$.

\section{PROOF OF THEOREM 3}

The arguments which we shall use are the same as in the proof of Proposition 3. Let $S_{n}$ be the symmetric group of degree $n \geq 2$, and suppose that $p$ is a prime divisor of $n$. Let $R$ be a field of characteristic $p$ and $A_{m n}^{S_{n}}$ be the algebra of vector invariants over $R$ with respect to $S_{n}$. Let

$$
v_{s_{1}, \ldots, s_{m}}, \quad s_{1}+\ldots+s_{m} \geq 1
$$

denote a polynomial in $A_{m n}^{S_{n}}$ of the form

$$
v_{s_{1}, \ldots, s_{m}}=\sum_{j=1}^{n} x_{1 j}^{s_{1}} \ldots x_{m j}^{s_{m}} .
$$

Recall that every vector invariant $v \in A_{m n}^{S_{n}}$ is an $R$-linear combination of the invariants

$$
w=\sum_{u \in\left\{\sigma(f) \mid \sigma \in S_{n}\right\}} u,
$$

where $f$ varies over the monomials in $A_{m n}$ which appear in $v$.

To prove Theorem 3 , it is sufficient to show that if $p^{\alpha} \mid n$, then every system of $R$-algebra generators of $A_{m n}^{S_{n}}$ contains at least one generator $v$ of degree $m\left(p^{\alpha}-1\right)$. The crucial point is that the invariant $v_{p^{\alpha}-1, \ldots, p^{\alpha}-1}$ of degree $m\left(p^{\alpha}-1\right)$ cannot be presented as a polynomial over $R$ in vector invariants of smaller degree. 
We denote by $W_{m n}^{S_{n}}$ the set of $R$-algebra generators of $A_{m n}^{S_{n}}$ of the form

$$
w=\sum_{u \in\left\{\sigma(f) \mid \sigma \in S_{n}\right\}} u,
$$

where $f$ is a monomial in $A_{m n}$ of degree less than $m\left(p^{\alpha}-1\right)$. Since every vector invariant $v \in A_{m n}^{S_{n}}$ whose degree is less than $m\left(p^{\alpha}-1\right)$ can be written as an $R$-linear combination of elements $w \in W_{m n}^{S_{n}}$, it suffices to prove that the invariant $v_{p^{\alpha}-1, \ldots, p^{\alpha-1}}$ is not representable as a polynomial over $R$ in the elements $w \in W_{m n}^{S_{n}}$. Let $l$ denote the cardinality of $W_{m n}^{S_{n}}$. We enumerate the elements of $W_{m n}^{S_{n}}$ by the numbers $1,2, \ldots, l$ and assume, for the contradiction, that the invariant $v_{p^{\alpha}-1, \ldots, p^{\alpha-1}}$ is a polynomial over $R$ in $w_{1}, \ldots, w_{l}$, that is,

$$
v_{p^{\alpha}-1, \ldots, p^{\alpha}-1}=\sum_{M} a_{\mu_{1}, \ldots, \mu_{l}} w_{1}^{\mu_{1}} \ldots w_{l}^{\mu_{l},} \quad a_{\mu_{1}, \ldots, \mu_{l}} \in R
$$

where $M$ is the set of all non-negative integers $\mu_{1}, \ldots, \mu_{l}$ such that

$$
\begin{aligned}
0 \leq \mu_{1}+\ldots+\mu_{l} & \leq m\left(p^{\alpha}-1\right), \\
\mu_{1} \operatorname{deg} w_{1}+\ldots+\mu_{l} \operatorname{deg} w_{l} & =m\left(p^{\alpha}-1\right) .
\end{aligned}
$$

Comparing the degrees of the monomials which appear in both sides of the last identity (with respect to each of the variables $x_{11}, \ldots, x_{m 1} ; \ldots ; x_{1 n}, \ldots, x_{m n}$ ), we find that $\mu_{k} \in$ $\left\{0,1, \ldots, p^{\alpha}-1\right\}, 1 \leq k \leq l$, and $\mu_{1}+\ldots+\mu_{l}>1$; moreover, every invariant

$$
w_{k}=\sum_{u \in\left\{\sigma\left(f_{k}\right) \mid \sigma \in S_{n}\right\}} u,
$$

which appears in the right-hand side with a non-zero coefficient, is generated by a monomial $f_{k} \in A_{m n}$ of the form

$$
f_{k}=\left(x_{1 j_{11}^{(k)}} \ldots x_{1 j_{1 v_{1}}^{(k)}}\right) \ldots\left(x_{m j_{m 1}^{(k)}} \ldots x_{m j_{m v_{m}}^{(k)}}\right)
$$

where $1 \leq j_{i j_{i 1}}^{(k)} \leq \ldots \leq j_{i v_{i}^{(k)}} \leq n$ and $j_{i v_{i}^{(k)}} \leq p^{\alpha}-1$ at least for one $i, i=1,2, \ldots, m$.

We denote by $\omega_{k}$ the value of $w_{k}$ at the point

$$
\left(x_{11}, \ldots, x_{m 1} ; \ldots ; x_{1 n}, \ldots, x_{m n}\right)=(1, \ldots, 1 ; \ldots ; 1, \ldots, 1)
$$

and observe that $\omega_{k}=\left|\operatorname{orb}\left(f_{k}\right)\right|$, where

$$
\operatorname{orb}\left(f_{k}\right)=\left\{\sigma\left(f_{k}\right) \mid \sigma \in S_{n}\right\}
$$

is the orbit of $f_{k}$ under $S_{n}$. If

$$
S_{n}\left(f_{k}\right)=\left\{\sigma \in S_{n} \mid \sigma\left(f_{k}\right)=f_{k}\right\}
$$

is the isotropy group of $f_{k}$, then

$$
\left|\operatorname{orb}\left(f_{k}\right)\right|=\frac{\left|S_{n}\right|}{\left|S_{n}\left(f_{k}\right)\right|}=\frac{n !}{\left|S_{n}\left(f_{k}\right)\right|} .
$$


Since $j_{i v_{i}^{(k)}} \leq p^{\alpha}-1$ for every $k=1,2, \ldots, l$ and at least for one $i, i=1,2, \ldots, m$, the exponent of $p$ in the prime factorisation of every $\left|S_{n}\left(f_{k}\right)\right|$ is less than the exponent of $p$ in the prime factorisation of $n$ !. This implies that $\left|\operatorname{orb}\left(f_{k}\right)\right|$ is divisible by $p$, therfore $\omega_{k}=0$ in $R$ for all $k=1,2, \ldots, l$. Differentiating now identity (3) with respect to $x_{11}$, setting

$$
x_{11}=\ldots=x_{m 1}=\ldots=x_{1 n}=\ldots=x_{m n}=1
$$

in the resulting identity, and taking into account that every product

$$
w_{1}^{\mu_{1}} \ldots w_{l}^{\mu_{l}}
$$

in the right-hand side of (3) involves at least two factors, say $w_{k}$ and $w_{k^{\prime}}$, with $1 \leq k \leq k^{\prime} \leq l$, we arrive at the relation $p-1=0$, which is impossible in $R$.

Since the invariant $v_{p^{\alpha}-1, \ldots, p^{\alpha}-1}$ cannot be written as a polynomial over $R$ in vector invariants of smaller degree, every system of $R$-algebra generators of $A_{m n}^{S_{n}}$ has to contain at least one generator of degree $m\left(p^{\alpha}-1\right)$. Observing now that every system of $R$-algebra generators of $A_{m n}^{S_{n}}$ contains a generator of degree $n$ (for example, the invariant $w=x_{11} \ldots x_{1 n}$ ), we find that it has to contain a generator $v$ whose degree is no less than $\max \left\{n, m\left(p^{\alpha}-1\right)\right\}$.

\section{PROOF OF COROLLARY 3}

Let $R$ be a commutative ring with the unit element 1 , let $p$ be a prime divisor of $n !$, and $\mathfrak{m}$ be a maximal ideal in $R$ that contains $p$. Then $F=R / \mathfrak{m}$ is a field of characteristic $p$. Consider the reduction homomorphism

$$
\varphi: R\left[x_{11}, \ldots, x_{m 1} ; \ldots ; x_{n 1}, \ldots, x_{m n}\right] \rightarrow F\left[x_{11}, \ldots, x_{m 1} ; \ldots ; x_{n 1}, \ldots, x_{m n}\right],
$$

which leaves fixed all the variables $x_{i j}, 1 \leq i \leq m, 1 \leq j \leq n$. This homomorphism induces a surjective homomorphism

$$
\psi: R\left[x_{11}, \ldots, x_{m 1} ; \ldots ; x_{n 1}, \ldots, x_{m n}\right]^{S_{n}} \rightarrow F\left[x_{11}, \ldots, x_{m 1} ; \ldots ; x_{1 n}, \ldots, x_{m n}\right]^{S_{n}} .
$$

Therefore $\psi$ maps every set of generators of $R\left[x_{11}, \ldots, x_{m 1} ; \ldots ; x_{1 n}, \ldots, x_{m n}\right]^{S_{n}}$ to a set of generators of $F\left[x_{11}, \ldots, x_{m 1} ; \ldots ; x_{1 n}, \ldots, x_{m n}\right]^{S_{n}}$. This fact and Corollary 2 imply that every system of $R$-algebra generators of $R\left[x_{11}, \ldots, x_{m 1} ; \ldots ; x_{1 n}, \ldots, x_{m n}\right]^{S_{n}}$ contains a generator of degree at least $\max \{n, m(p-1)\}$. In particular, if $n=p$ is a prime number, we conclude that it contains a generator whose degree is no less than $\max \{n, m(n-1)\}$.

\section{REFERENCES}

1. D. J. Benson, Polynomial Invariants of Finite Groups. Cambridge Univ. Press, Cambridge, 1993.

2. N. Bourbaki, Elements of Mathematics, Algebra II. Springer, Berlin, 1990.

3. H. E. A. Campbell, I. Hughes, and R. D. Pollack, Vector invariants of symmetric groups, Canad. Math. Bull. (1990) 33, 391-397.

4. J. A. Dieudonne and J. B. Carrel, Invariant Theory, Old and New. Academic Press, New York, 1971. 
5. P. Fleischmann, A new degree bound for vector invariants of symmetric groups. Trans. Amer. Math. Soc. (1998) 350, 1703-1712.

6. D Hilbert, Über die vollen Invariantensystem. Math. Ann. (1893) 42, 313-373.

7. G. Kemper, Lower degree bounds for modular invariants and a question of I. Hughes. Transformation Groups (1998) 3, 135-144.

8. E. Noether, Der Endlichkeitssatz der Invarianten endlicher Gruppen. Math. Ann. (1916) 77, 8992.

9. E. Noether, Der Endlichkeitssatz der Invarianten endlicher linearer Gruppen der Charakteristik p. Nachr. Ges. Wiss. Göttingen (1926), 28-35.

10. D. R. Richman, Invariants of finite groups over fields of characteristic p. Adv. Math. (1996) 124, $25-48$.

11. D. R. Richman, Explicit generators of the invariants of finite groups. Adv. Math. (1996) 124, 49-76.

12. L. Smith, Polynomial Invariants of Finite Groups. A K Peters, Wellesley, MA, 1995.

13. L. Smith, Polynomial invariants of finite groups. A survey of recent developments. Bull. Amer. Math. Soc. (1997) 34, 211-250.

14. S. A. Stepanov, Transcendence bases of the algebra of vector invariants for a symmetric group. Proc. Intern. Conf. Number Theory, Berlin, 1999, 487-501.

15. S. A. Stepanov, Polynomial invariants of finite groups in prime characteristic. Discrete Math. Appl. (1999) 9, 343-354.

16. E. Waring, Meditationes Algebraicae. Cambridge Univ. Press, Cambridge, 1782.

17. H. Weyl, The Classical Groups, their Invariants and Representations. New Jersey, 1939. 